\title{
Monopole condensation in two-flavour Adjoint QCD
}

\author{
Giuseppe Lacagnina* \\ Dipartimento di Fisica and INFN, Pisa, Italy \\ E-mail: lacagnina@df.unipi.it
}

\section{Guido Cossu}

Scuola Normale Superiore and INFN, Pisa, Italy

E-mail: g.cossuesns.it

\section{Massimo D’Elia}

Dipartimento di Fisica and INFN, Genova, Italy

E-mail: Massimo.Delia@ge.infn.it

\section{Adriano Di Giacomo, Claudio Pica}

Dipartimento di Fisica and INFN, Pisa, Italy

E-mail: digiaco@df.unipi.it, pica@df.unipi.it

Two distinct phase transitions occur at different temperatures in QCD with adjoint fermions (aQCD): deconfinement and chiral symmetry restoration. In this model, quarks do not explicitly break the center Z(3) symmetry and therefore the Polyakov loop is a good order parameter for the deconfinement transition. We study monopole condensation by inspecting the expectation value of an operator which creates a monopole. Such a quantity is expected to be an order parameter for the deconfinement transition as in the case of fundamental fermions.

XXIV International Symposium on Lattice Field Theory

July 23-28 2006

Tucson Arizona, US

${ }^{*}$ Speaker. 


\section{Introduction}

As lattice simulations seem to show, chiral symmetry restoration and deconfinement occur for QCD at the same temperature, making it extremely hard to disentangle them. On the other hand, QCD with quarks in the adjoint representation of $S U(3)$ (aQCD) is a model in which two transitions take place at different temperatures [1]. In aQCD, the $Z(3)$ center symmetry is not broken by adjoint fermions and therefore the Polyakov loop is an order parameter even at finite quark mass as in the pure gauge case.

The authors of ref.[1] find on the lattice two distinct phase transitions; they observe a first order deconfinement transition and a continuous chiral transition. They also check that the observables which are sensitive to deconfinement are not significantly affected by the chiral transition.

Another way to study confinement consists in looking for magnetic charge condensation, which signals dual superconductivity of the vacuum $[2,3,4,5]$. In this case, one constructs an operator which carries magnetic charge and looks at its vacuum expectation value, which is expected to be different from zero in the confined phase and strictly zero in the deconfined phase. Our goal is to study monopole condensation in aQCD in order to check that it is unaffected by the chiral transition.

In this report we present the results of a lattice simulation of aQCD in which we started our investigation; it has to be considered as work in progress. The qualitative behaviour of the monopole order parameter was found to be consistent with the expectations; however, for time limitations, we could not simulate quark masses that were light enough to study its chiral properties. We are currently continuing our investigation in this direction. We also need to understand the scaling properties of the monopole condensation parameter.

\section{1 aQCD}

Quarks in the adjoint representation of QCD have 8 colour degrees of freedom and can be described with $3 \times 3$ hermitian traceless matrices

$$
Q(x)=Q^{a}(x) \lambda_{a}
$$

using Gell-Mann's $\lambda$ matrices. In order to write the fermionic part of action for this model, the 8 -dimensional representation of the gauge links (which is real) must be used:

$$
U_{(8)}^{a b}=\frac{1}{2} \operatorname{Tr}\left(\lambda^{a} U_{(3)} \lambda^{b} U_{(3)}^{\dagger}\right)
$$

The full action is therefore given by

$$
S=S_{G}\left[U_{(3)}\right]+\sum_{x, y} \bar{Q}(x) M\left(U_{(8)}\right)_{x, y} Q(y)
$$

where $S_{G}$ is the usual $S U(3)$ gauge action and $M$ is the fermionic matrix. The Polyakov loop is defined as in the $S U(3)$ Yang Mills case

$$
L_{3} \equiv\left\langle\frac{1}{3 L_{s}^{3}}\left|\sum_{\vec{x}} \operatorname{Tr} \prod_{x_{0}=1}^{L_{t}} U_{0}^{(3)}\left(x_{0}, \vec{x}\right)\right|\right\rangle
$$


This quantity is an order parameter for the spontaneous breaking of the center symmetry and is related to the free energy of isolated static fundamental charge.

\subsection{Monopole condensation}

A magnetically charged operator is constructed in such a way that it adds a monopole field to a given configuration. Its expectation value is given by

$$
\langle\mu\rangle=\frac{\tilde{Z}}{Z}
$$

where $\tilde{Z}$ is the partition function for the action in presence of a monopole. To better cope with fluctuations, one instead calculates the quantity

$$
\rho=\frac{d}{d \beta} \ln \langle\mu\rangle=\langle S\rangle_{S}-\langle\tilde{S}\rangle_{\tilde{S}}
$$

in which $\langle\tilde{S}\rangle_{\tilde{S}}$ is the average of the action with a monopole insertion weighted with the modified action itself. Therefore, two simulations have to be run for each value of $\beta$. The parameter $\langle\mu\rangle$ should be different from zero in the confined phase where magnetic charges condense and drop to zero at deconfinement where magnetic symmetry is restored. This drop corresponds to a negative peak of $\rho$. In the vicinity of the critical temperature, $\beta \simeq \beta_{c}, \rho$ is expected to scale as

$$
\rho L^{-1 / v}=f\left(L^{1 / v}\left(\beta_{c}-\beta\right)\right)
$$

The critical exponent $v$ should be equal to $1 / 3$ for a first order transition.

\section{Simulation details}

We have simulated two flavours of adjoint staggered fermions using the RHMC algorithm [6]. Trajectories had a length of $N_{M D} \delta t=1$, and an integration step of $\delta t=0.02$. Acceptance was above $90 \%$. Inversions of the fermionic matrix were performed with the Conjugate Gradient algorithm.

We have run simulations with two different lattice sizes, i.e. $L_{s}^{3} \times L_{t}=12^{3} \times 4,16^{3} \times 4$. We have calculated the chiral condensate, the Polyakov loop and the average plaquette for 12 values of $\beta$ in the range $(3.0,7.0)$. We computed the average plaquette and the $\rho$ parameter. For time constraints, we were limited to runs at a bare quark mass of $a m=0.04$. In order to simulate an action with a monopole contribution, $C^{*}$ boundary conditions had to be implemented [7].

Our code has been run on the ApeMille machine in Pisa and the ApeNEXT facility in Rome.

\section{Results}

For each value of $\beta$, we have calculated the Polyakov loop and the $\rho$ parameter on both volumes, $12^{3} \times 4,16^{3} \times 4$. For the Polyakov loop, we reproduced the results of [1] at the same value of the bare quark mass, $a m=0.04$, see Figure (2). Figure (1) shows the results for the $\rho$ parameter. The position of the negative peak shifts slightly with the volume as one would expect for a physical 


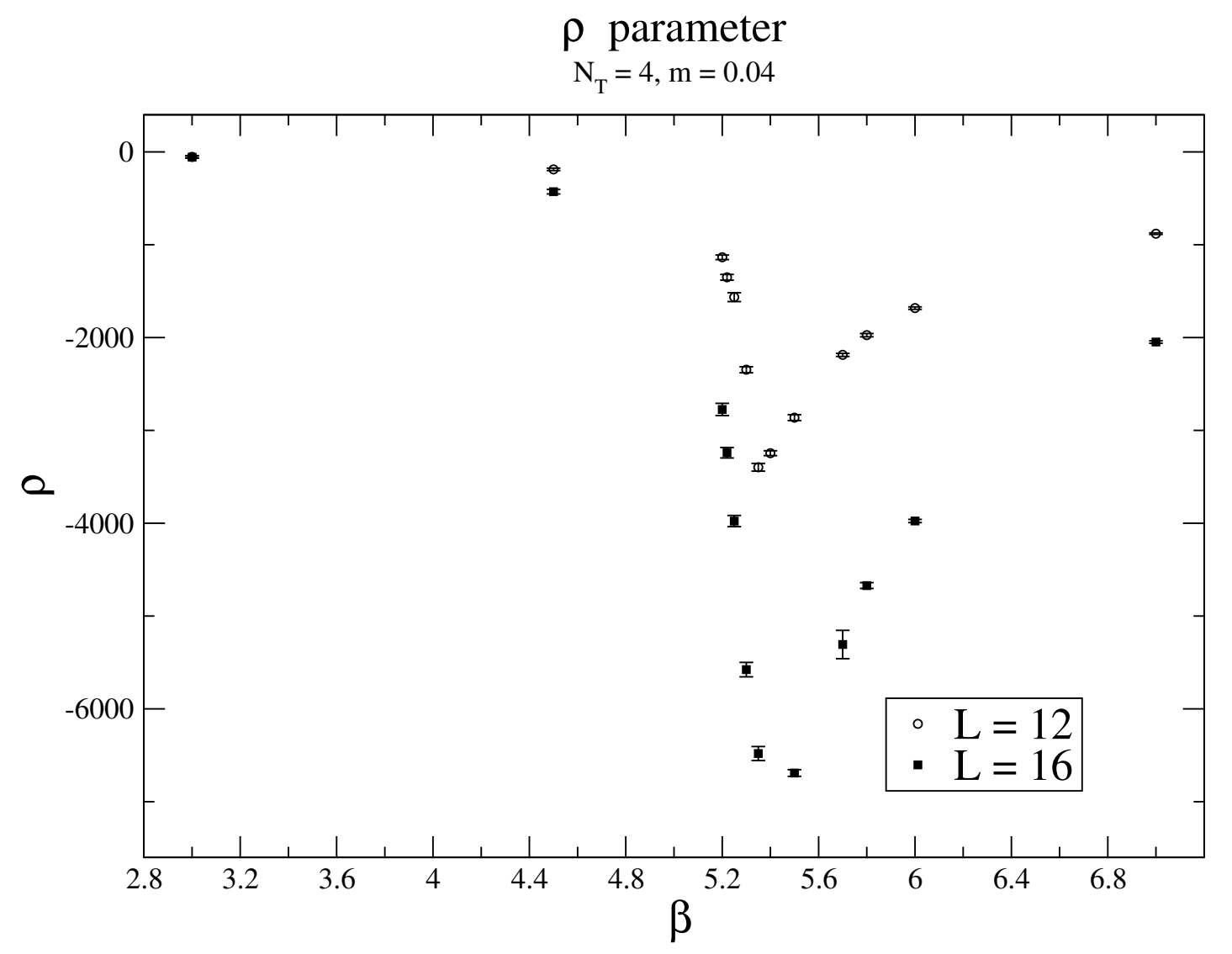

Figure 1: The $\rho$ parameter, with $a m=0.04, L_{t}=4$, for two different spatial volumes

transition. This estimate of the pseudocritical coupling is consistent with the position of the jump in the Polyakov loop. Furthermore, as $\beta \rightarrow 0$, one expects $\rho$ to become volume independent, as $\mu$ should tend to a nonzero constant. Our results are compatible with this expectation. We are currently working on the analysis of the scaling properties of $\rho$. We could not study the behaviour of $\rho$ at the chiral transition as the bare quark mass turned out to be too large to see any visible effect in the chiral condensate susceptibility.

\section{Conclusions}

We have studied monopole condensation in lattice QCD with $N_{f}=2$ staggered fermions in the adjoint representation. We evaluated the expectation value of a magnetically charged operator, which is expected to be an order parameter for confinement. For this operator, we observed a qualitative agreement with the expected behaviour. In particular, the data were consistent with the theoretical predictions for the $\beta \rightarrow 0$ limit: $\rho$ becomes volume independent. We are currently working on simulations with lighter quark masses and larger spatial volumes, in order to study the behaviour of the $\rho$ parameter at the chiral transition and its scaling properties. 


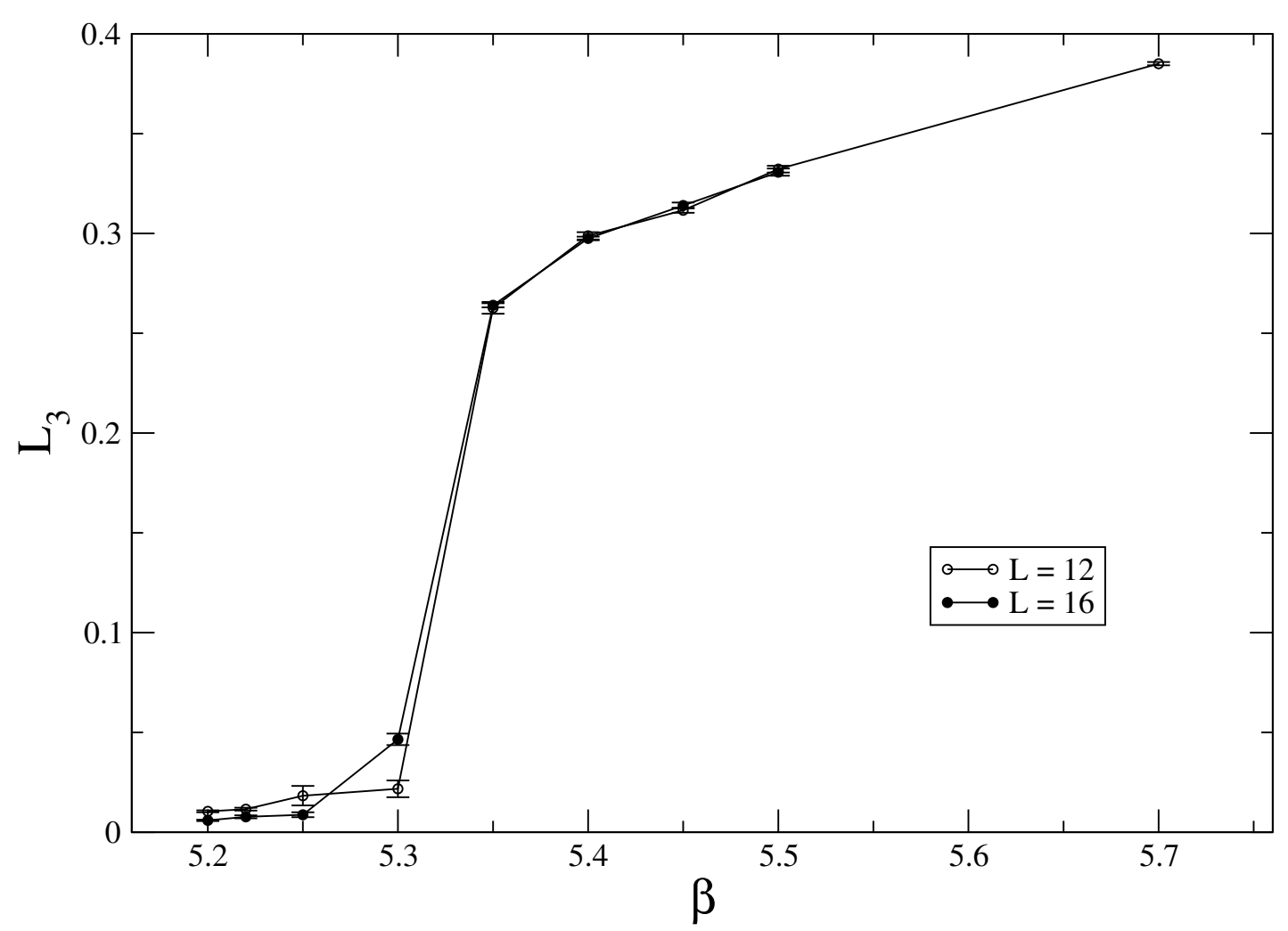

Figure 2: The Polyakov loop, with $a m=0.04, L_{t}=4$, for two different spatial volumes. Lines are left to guide the eye.

\section{References}

[1] F. Karsch, M. Lutgemeier, Nucl. Phys. B 550, 449 (1999), hep-lat/9812023

[2] A. Di Giacomo, B. Lucini, L. Montesi, G. Paffuti, Phys. Rev. D 61, 034503 (2000), hep-lat/9906024

[3] A. Di Giacomo, B. Lucini, L. Montesi, G. Paffuti, Phys. Rev. D61, 034504 (2000), hep-lat/9906025

[4] J. M. Carmona, M. D’Elia, A. Di Giacomo, B. Lucini, G. Paffuti, Phys. Rev. D64, 114507 (2001), hep-lat/0103005

[5] M. D’Elia, A. Di Giacomo, B. Lucini, G. Paffuti, C. Pica, Phys. Rev. D71, 114502 (2005), hep-lat/0503035

[6] M. A. Clark, A. D. Kennedy, Nucl. Phys. Proc. Suppl. 129, 850 (2004), hep-lat/0309084

[7] J. M. Carmona, M. D’Elia, A. Di Giacomo, B. Lucini, Int. J. Mod. Phys. C11, 637 (2000), hep-lat/0003002 